\title{
The relationship between mean arterial pressure and decreased glomerular filtration rate in rural areas of Northeast China
}

Hongmei Yang ${ }^{1}$, Xiaofan Guo ${ }^{1}$, Xingang Zhang ${ }^{1}$, Zhao Li ${ }^{1}$, Shasha Yu ${ }^{1}$, Liqiang Zheng ${ }^{2}$, Wenna Li ${ }^{1}$, Ying Zhou ${ }^{1}$ and Yingxian Sun ${ }^{1 *}$

\begin{abstract}
Background: Low mean arterial pressure (MAP) can cause low renal blood flow and damage the kidneys. However, in the general population, it remains unclear whether or not decline in renal function is related to MAP. The present study examined the relationship between MAP and decreased glomerular filtration rate(GFR) in participans aged $\geq 35$ years from the Liaoning province of China.

Methods: A total of 11345 representative individuals aged $\geq 35$ years was selected and a cross-sectional survey was conducted from January 2012 to August 2013 to describe the gender-specific prevalence and factors associated with decreased GFR in rural areas of Liaoning Province.

Results: Men with decreased eGFR (eGFR $<60 \mathrm{ml} / \mathrm{min}$ per $1.73 \mathrm{~m}^{2}$ ) were older, and had higher meanWC, systolic and diastolic BP, PP, MAP, total fasting glucose, LDL-C, glyceride and uric acid levels and were current drinker/ smoker at the baseline (all $\mathrm{P}<0.05$ ). Those with low education level, low income, low physical activity, low hemoglobin and HDL-C level had decreased eGFR (all $P<0.05$ ). In women, the results were similar to those of men, but DBP and drinking status had no associations with the eGFR at the baseline (all $P<0.05$ ). After adjustment for age, men with MAP of $>112.2 \mathrm{mmHg}$ versus $\leq 93.8 \mathrm{mmHg}$ had ORs for decreased eGFR of 2.367 (95 \% Cl: 1.248 to 4.488). After multivariable adjustment, an MAP of $>112.2 \mathrm{mmHg}$ versus $\leq 93.8 \mathrm{mmHg}$ had an OR for decreased eGFR of 3.249 (95 \% Cl:1.394 to 7.575) in men, whereas in women, MAP was not associated with decreased eGFR.

Conclusions: MAP was associated with decreased eGFR in men, while in women MAP was not associated with decreased eGFR. These findings provide some evidence that a different adaptive response to renal regulation may exist in males and females.
\end{abstract}

Keywords: Mean arterial pressure, Decreased glomerular filtration rate, Relationship, Rural areas

\section{Background}

Chronic kidney disease (CKD) is recognized as a global public health problem , and patients with CKD have a significantly increased risk of cardiovascular disease [1]. Mild renal insufficiency has been considered to be an independent risk factor for cardiovascular disease, and the correlation in people, in the absence of other cardiovascular risk factors has been confirmed[2]. Mean arterial pressure (MAP) is considered to be the perfusion

\footnotetext{
* Correspondence: sunyingxian12@126.com

${ }^{1}$ Department of Cardiology, The First Hospital of China Medical University, 155 Nanjing North Street, Heping District, Shenyang 110001, Liaoning, China Full list of author information is available at the end of the article
}

pressure for the organs of the body. Low MAP can cause low renal blood flow (RBF) and damage the kidney [3]. However, this doesn't mean that RBF increases as the MAP increases [4-7].

Recent interest has been aroused in the relationship of renal damage with arterial stiffness evaluated by the augmentation index (AIx) and blood pressure including central blood pressure (cBP) and peripheral blood pressure (pBP). Several studies have investigated the association of AIx, cBP and pBP with glomerular filtration rate (GFR), but they have obtained inconsistent results [8-11]. How MAP affects kidney function and gender differences in this mechanism remains unclear.
C Biomed Central 
In the general population, is unknown how MAP influences the GFR? This study examined the relation between MAP and decreased GFR in participants aged $\geq$ 35 years from the Liaoning province of China. Further, we studied the relationship to see if there was a difference between the sexes.

\section{Methods}

\section{Study population}

The study was approved by the Ethics Committee of China Medical University (Shenyang, China). Liaoning Province is located in Northeast China. A representative sample of people aged $\geq 35$ years was selected, and a cross-sectional survey was conducted from January 2012 to August 2013 to describe the sex-specific prevalence and factors associated with decreased GFR in rural areas of Liaoning Province. The study adopted a multi-stage, stratified random cluster-sampling scheme. In the first stage, three counties (Dawa, Zhangwu, and Liaoyang County) were selected from the eastern, southern, and northern regions of Liaoning province. In the second stage, one town was randomly selected from each county (a total of three towns). In the third stage, six to eight rural villages near each town were randomly selected (a total of 26 rural villages). Exclusion criteria for individuals in the present study included pregnancy, malignant tumor, or mental disorder. All the eligible permanent residents aged $\geq 35$ years from each village were invited to join the study (a total of 14016 participants). Of those, 11956 participants responded and completed the study: the response rate was $85.3 \%$. All procedures were performed in accordance with standard ethical principles. Written consent was obtained from all participants after they had been informed of the objectives, benefits, medical procedures and the confidentiality of personal information. For illiterate participants, we obtained written informed consent from their proxies. In this report, we used data obtained at baseline, and only participants with a complete set of data for the variables analyzed in the study were included, making a final sample size of 11345 (5253 men and 6092 women).

\section{Data collection and measurements}

Data were collected during a single clinic visit by trained doctors and nurses using a standard questionnaire by face-to-face interview. Before the survey was performed, all eligible investigators were invited to attend training. The training content included the purpose of this study, how to administer the questionnaire, the standard methods of measurement, the study procedures, and the importance of standardization. After the training a strict test was carried out, and only those who scored perfectly on the test could become investigators. During data collection, our inspectors received further instructions and support. There was a central steering committee with a subcommittee for quality control.

Data on demographic characteristics, lifestyle risk factors, dietary habits, family income, family history of cardiovascular disease and medical history of hypertension were obtained by interview with a standardized questionnaire. Educational level was divided into primary school or below, middle school, and high school or above. Family income was classified as $\leq 5000,5000-20000$ and >20 000 $\mathrm{CNY} /$ year.

According to the American Heart Association protocol, the investigators performed blood pressure (BP) measurement three times for each participant at 2-min intervals after at least 5 min of rest, using a standardized automatic electronic sphygmomanometer (HEM-907; Omron), which had already been validated according to the British Hypertension Society protocol [12]. The participants were advised to avoid caffeinated beverages and exercise for at least $30 \mathrm{~min}$ before the measurement. During the measurement, the participants were seated with the arm supported at the level of the heart. The average of three BP measures was calculated and used in all analyses.

Weight and height were measured to the nearest 0.1 $\mathrm{kg}$ and $0.1 \mathrm{~cm}$ respectively, with the participants in lightweight clothing and without shoes. Waist circumference (WC) was measured (to the nearest $0.1 \mathrm{~cm}$ ) at the umbilicus using a non-elastic tape, with the participants standing, at the end of normal expiration. BMI was calculated as weight in kilograms divided by the square of the height in meters.

Fasting blood samples were collected from all participants in the morning after at least $12 \mathrm{~h}$ of fasting. Blood samples were obtained from an antecubital vein into Vacutainer tubes containing EDTA. Fasting plasma glucose (FPG), total cholesterol (TC), low-density lipoprotein cholesterol (LDL-C), high-density lipoprotein cholesterol (HDL-C), triglyceride (TG), serum creatinine (Scr) and other routine blood biochemical indexes were analyzed enzymatically on an autoanalyzer. All laboratory equipment was calibrated, and blinded duplicate samples were used.

\section{Definitions}

According to the JNC-8 report [13], hypertension is defined as SBP $\geq 140 \mathrm{mmHg}$ and/or DBP $\geq 90 \mathrm{mmHg}$ and/or the use of antihypertensive medications. According to the World Health Organization (WHO) criteria, BMI was categorized into three groups: normal (BMI $<25 \mathrm{~kg} / \mathrm{m} 2)$, overweight $(25 \leq \mathrm{BMI}<30 \mathrm{~kg} / \mathrm{m} 2)$ and obesity (BMI $\geq 30 \mathrm{~kg} / \mathrm{m} 2$ ) [14]. Abdominal obesity was defined as $\mathrm{WC} \geq 88 \mathrm{~cm}$ for females and $\mathrm{WC} \geq 102 \mathrm{~cm}$ for males [15]. Dyslipidemia was defined according to the National Cholesterol Education Program-Third Adult Treatment Panel (ATP III) criteria [16]. High TC was 
defined as $\mathrm{TC} \geq 6.21 \mathrm{mmol} / \mathrm{L}(240 \mathrm{mg} / \mathrm{dL})$. Low HDL-C was defined as HDL-C $<1.03 \mathrm{mmol} / \mathrm{L}(40 \mathrm{mg} / \mathrm{dL})$. High LDL-C was defined as LDL-C $\geq 4.16 \mathrm{mmol} / \mathrm{L}(160 \mathrm{mg} / \mathrm{dL})$. High TG was defined as TG $\geq 2.26 \mathrm{mmol} / \mathrm{L}(200 \mathrm{mg} / \mathrm{dL})$. Dyslipidemia was defined as having at least one of the disorders mentioned above. Diabetes mellitus (DM) and anemia were both diagnosed according to the WHO criteria: DM was defined as FPG $\geq 7 \mathrm{mmol} / \mathrm{L}(126 \mathrm{mg} / \mathrm{dL})$ and/or being on treatment for diabetes; anemia was defined as a hemoglobin level $<13.0 \mathrm{~g} / \mathrm{dL}(<130 \mathrm{~g} / \mathrm{L})$ for men and $<12.0 \mathrm{~g} / \mathrm{dL}(<120 \mathrm{~g} / \mathrm{L})$ for women $[17,18]$. Hyperuricaemia was determined as uric acid $>422 \mu \mathrm{mol} / \mathrm{L}$ for men and $>363 \mu \mathrm{mol} / \mathrm{L}$ for women [19]. Drinking and smoking status were divided into current drinkers/smokers and nondrinkers/smokers. Physical activity included occupational and leisure-time physical activity. A detailed description of the methods has been presented before [20]. Occupational and leisure-time physical activity were merged and regrouped into three categories: (1) low-participants who reported light levels of both occupational and leisure-time physical activity; (2) moderateparticipants who reported moderate or high levels of either occupational or leisure-time physical activity; and (3) high-participants who reported a moderate or high level of both occupational and leisure-time physical activity.

The dietary pattern was assessed using recall of foods eaten in the previous year. First, the participants were questioned on their average consumption of several food items per week. The reported consumption was quantified approximately in terms of grams per week (vegetable consumption: rarely $=3,<1000 \mathrm{~g}=2,1000-2000 \mathrm{~g}=1, \geq 2000$ $\mathrm{g}=0$; meat consumption, including red meat, fish, and poultry: rarely $=0,<250 \mathrm{~g}=1,250-500 \mathrm{~g}=2, \geq 500 \mathrm{~g}=3$ ). Subsequently, a special diet score (vegetable consumption score plus meat consumption score) was calculated for each participant (range 0-6). Higher values of the diet score indicate higher meat consumption and lower vegetable consumption and greater adherence to a Westernized diet, while lower values indicate adherence to the Chinese diet. Similar methods for calculating diet score could be found in the ATTICA study [21].

\section{Assessment of glomerular filtration rate}

Fasting blood samples were collected as described under "Data collection and measurement". Serum creatinine (Scr) was measured enzymatically on an autoanalyzer. The GFR was estimated using an equation originating from the CKD Epidemiology Collaboration (CKD-EPI) equation [22], which is more appropriate than the Modification of Diet in Renal Disease (MDRD) Study group equation [23]. Decreased GFR was defined as estimated GFR (eGFR) $<60 \mathrm{ml} / \mathrm{min} / 1.73 \mathrm{~m} 2$.

\section{Statistical analysis}

Descriptive statistics were calculated for all the variables, including continuous variables (reported as mean values and standard deviations) and categorical variables (reported as numbers and percentages). Differences among them were evaluated using Student's t-test, ANOVA, non-parametric tests or the $\mathrm{X}^{2}$-test as appropriate. Multivariate logistic regression analyses were used to identify independent factors and associated comorbidities of decreased eGFR, with odds ratios (ORs) and corresponding $95 \%$ confidence intervals (CIs) calculated. All the statistical analyses were performed using SPSS version 17.0 software, and $\mathrm{P}$ values $<0.05$ were considered to be statistically significant.

\section{Results}

\section{Baseline characteristics of study population}

Table 1 presents baseline characteristics of the participants according to the eGFR categories and sex. Men with decreased eGFR (eGFR $<60 \mathrm{ml} / \mathrm{min}$ per $1.73 \mathrm{~m} 2$ ) had higher age, mean WC, systolic and diastolic BP, PP, MAP, total fasting glucose, LDL-C, glyceride and uric acid levels and were current drinker/smoker at the baseline (all $\mathrm{P}<0.05$ ). Those with low education level, low income, low physical activity, low hemoglobin and HDL-C level had decreased eGFR (all $\mathrm{P}<0.05$ ). In women, the results were similar to those of men, but DBP and drinking status had no association with the eGFR in women.

\section{The prevalence of decreased eGFR by quartiles of MAP in men and women}

Figure 1 shows that the prevalence of decreased eGFR by quartiles of MAP was different in men and women. For men, the prevalence of decreased eGFR increased as the MAP increased. The prevalence of decreased eGFR in men was lowest when $93.8 \leq \mathrm{MAP}<101.8 \mathrm{mmHg}$, while for women, when $98.4 \leq \mathrm{MAP}<109.1 \mathrm{mmHg}$, the prevalence of decreased eGFR was lower than in the group with lower MAP.

\section{The $\mathrm{OR}$ and $95 \% \mathrm{Cl}$ for the prevalence of decreased eGFR as a function of quartiles of MAP}

Figure 2 shows that after adjustment for age, that men with a MAP of $>112.2 \mathrm{mmHg}$ versus $\leq 93.8 \mathrm{mmHg}$ had an OR for decreased eGFR of 2.367 (95\% CI: 1.248 to 4.488).After multivariable adjustment, men with a MAP of $>112.2 \mathrm{mmHg}$ versus $\leq 93.8 \mathrm{mmHg}$ had an OR for decreased eGFR of 3.249 (95\% CI: 1.394 to 7.575 ). In women, MAP was not associated with decreased eGFR.

\section{Discussion}

This is, to our knowledge, the first study to compare the association of MAP with decreased eGFR in a large group of participants. In this study, we demonstrated 
Table 1 Characteristics of subjects with eGFR $\geq 60$ or eGFR $<60$ by different genders

\begin{tabular}{|c|c|c|c|c|c|c|}
\hline \multirow[b]{2}{*}{ Characteristics } & \multicolumn{3}{|l|}{ Male } & \multicolumn{3}{|l|}{ Female } \\
\hline & $\mathrm{eGFR} \geq 60$ & eGFR $<60$ & $P$ value & $\mathrm{eGFR} \geq 60$ & eGFR $<60$ & $P$ value \\
\hline $\mathrm{N}$ & 5165 & 88 & & 5939 & 153 & \\
\hline Age (year) & $54.13 \pm 10.64$ & $68.86 \pm 10.46$ & $<0.001$ & $52.97 \pm 10.05$ & $68.73 \pm 8.96$ & $<0.001$ \\
\hline MeanWC & $83.71 \pm 9.70$ & $87.03 \pm 10.34$ & 0.004 & $81.17 \pm 9.69$ & $84.07 \pm 10.30$ & $<0.001$ \\
\hline BMI (kg/m2) & $24.72 \pm 3.54$ & $25.34 \pm 3.77$ & 0.168 & $24.85 \pm 3.75$ & $24.79 \pm 3.91$ & 1 \\
\hline Average SBP $(\mathrm{mmHg})$ & $143.30 \pm 22.41$ & $161.31 \pm 26.31$ & $<0.001$ & $139.68 \pm 23.80$ & $154.23 \pm 26.29$ & $<0.001$ \\
\hline Average DBP (mmHg) & $83.66 \pm 11.73$ & $89.04 \pm 16.33$ & 0.002 & $80.49 \pm 11.41$ & $82.51 \pm 14.78$ & 0.11 \\
\hline $\mathrm{PP}(\mathrm{mmHg})$ & $59.63 \pm 16.34$ & $72.27 \pm 21.71$ & $<0.001$ & $59.19 \pm 17.81$ & $71.72 \pm 20.84$ & $<0.001$ \\
\hline MAP $(\mathrm{mmHg})$ & $103.54 \pm 14.14$ & $113.13 \pm 17.43$ & $<0.001$ & $100.22 \pm 14.32$ & $106.42 \pm 16.71$ & $<0.001$ \\
\hline Race group (han, \%) & $4891,94.7$ & $85,96.6$ & 0.43 & $5633,94.8$ & 150,98 & 0.076 \\
\hline Education & & & $<0.001$ & & & $<0.001$ \\
\hline Primary school or below (n, \%) & $2139,41.4$ & $55,62.5$ & & 3325,56 & $133,86.9$ & \\
\hline Middle school (n, \%) & $2433,47.1$ & $26,29.5$ & & $2146,36.1$ & $18,11.8$ & \\
\hline High school or above (n, \%) & $593,11.5$ & $7,8.0$ & & $468,7.9$ & $2,1.3$ & \\
\hline Income (CNY/year) & & & $<0.001$ & & & $<0.001$ \\
\hline$<=5000(\mathrm{n}, \%)$ & 669,13 & $28,31.8$ & & $668,11.2$ & $39,25.5$ & \\
\hline $5000-20000(n, \%)$ & $2780,53.8$ & $43,48.9$ & & $3293,55.4$ & $77,50.3$ & \\
\hline$>20000(n, \%)$ & $1716,33.2$ & $17,19.3$ & & $1978,33.3$ & $37,24.2$ & \\
\hline Current drinking status (n, \%) & $2376,46.0$ & $13,14.8$ & $<0.001$ & $173,2.9$ & $3,2.0$ & 0.804 \\
\hline Current smoking status ( $\mathrm{n}, \%)$ & $2976,57.6$ & $26,29.5$ & $<0.001$ & $961,16.2$ & $44,28.8$ & $<0.001$ \\
\hline History of stroke $(n, \%)$ & $458,8.9$ & 29,33 & $<0.001$ & $480,8.1$ & $29,19.0$ & $<0.001$ \\
\hline History of heart disease $(n, \%)$ & $530,10.3$ & $28,31.8$ & $<0.001$ & $914,18.4$ & $63,41.2$ & $<0.001$ \\
\hline Physical activity & & & $<0.001$ & & & $<0.001$ \\
\hline Mild (n, \%) & $1132,21.9$ & $51,58.0$ & & $2092,35.2$ & $99,64.7$ & \\
\hline Moderate (n, \%) & $3746,72.5$ & $35,39.8$ & & $154,59.2$ & $41,26.8$ & \\
\hline High (n, \%) & $287,5.6$ & $2,2.3$ & & $332,5.6$ & $13,8.5$ & \\
\hline \multicolumn{7}{|l|}{ Laboratory parameters } \\
\hline Fasting glucose (mmol/L) & $5.94 \pm 1.65$ & $6.58 \pm 2.58$ & 0.013 & $5.85 \pm 1.58$ & $6.54 \pm 2.26$ & $<0.001$ \\
\hline Total cholesterol (mmol/L) & $5.16 \pm 1.03$ & $5.45 \pm 1.47$ & 0.109 & $5.28 \pm 1.09$ & $5.98 \pm 1.70$ & $<0.001$ \\
\hline Triglyceride $(\mathrm{mmol} / \mathrm{L})$ & $1.66 \pm 1.67$ & $1.86 \pm 1.59$ & 0.005 & $1.60 \pm 1.32$ & $2.24 \pm 1.75$ & $<0.001$ \\
\hline LDL-C (mmol/L) & $2.87 \pm .079$ & $3.12 \pm 1.01$ & 0.03 & $2.96 \pm 0.83$ & $3.33 \pm 1.14$ & $<0.001$ \\
\hline $\mathrm{HDL}-\mathrm{C}(\mathrm{mmol} / \mathrm{L})$ & $1.41 \pm 0.42$ & $1.21 \pm 0.27$ & $<0.001$ & $1.41 \pm 0.34$ & $1.35 \pm 0.41$ & 0.002 \\
\hline Hemoglobin (g/L) & $148.95 \pm 17.96$ & $138.89 \pm 20.56$ & $<0.001$ & $130.22 \pm 14.50$ & $125.75 \pm 16.14$ & $<0.001$ \\
\hline Uric acid & $331.85 \pm 81.25$ & $447.51 \pm 122.02$ & $<0.001$ & $252.94 \pm 64.55$ & $362.89 \pm 94.43$ & $<0.001$ \\
\hline
\end{tabular}

Data are expressed as the mean \pm SD or as $\mathrm{n}(\%)$

BMI Body mass index, WC Waist circumference, CNY Chinese Yuan (1 CNY = 0.161 USD), SBP Systolic blood pressure, DBP Diastolic blood pressure, LDL-C Low-density lipoprotein cholesterol, HDL-C High-density lipoprotein cholesterol

that MAP was associated with decreased eGFR in males. However, this association is not linear, from the lowest level of MAP to the highest level, but is significant only when MAP is $>112.2 \mathrm{mmHg}$. It is well known that BP is characterized by its pulsatile and steady components. The pulsatile component, estimated by PP, represents BP variation and is affected by left ventricular ejection fraction, large-artery stiffness, early pulse wave reduction, and pulse rate $[24,25]$. The steady component, estimated by MAP, is a function of left ventricular contractility, pulse rate, and vascular resistance and elasticity, averaged over time [26]. Adequate MAP is required to protect renal function, because below a certain MAP threshold, when autoregulation is exceeded, RBF decreases and this leads to acute renal injury [27]. When MAP does not exceed a certain level, there is no significant effect on renal function. With increasing MAP, the vascular resistance and elasticity increase, the arteries 


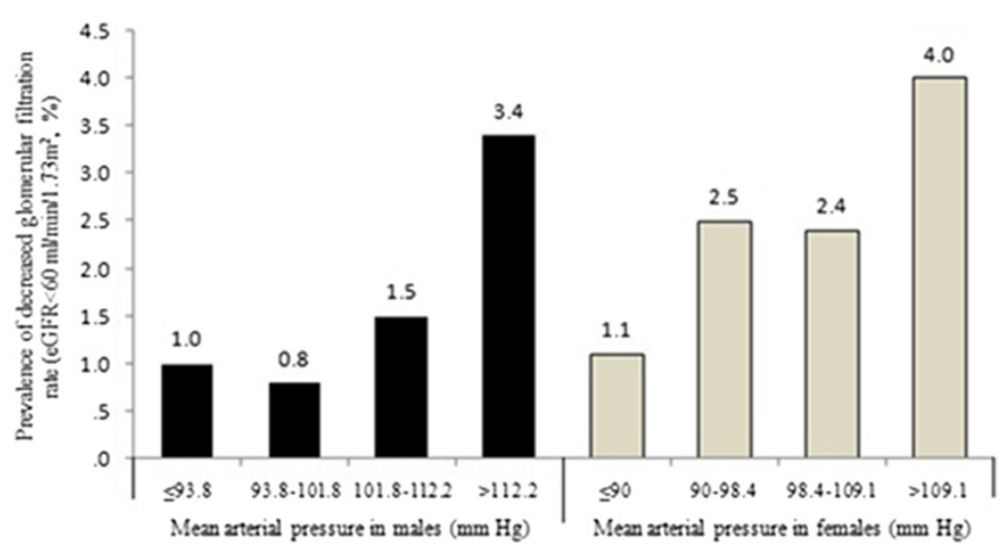

Fig. 1 The prevalence of decreased eGFR by quartiles of MAP in men and women

become progressively more stiff, and finally eGFR is decreased.

In our study, there were significant differences between men and women: in the latter, MAP was not associated with decreased eGFR. The trend of the prevalence of decreased eGFR as a function of quartiles of MAP appeared as a U-shaped curve. Recent studies have indicated that sex hormones play important roles in regulating the structure and/or function of the kidney, causing sex differences in a variety of characteristics [28-30]. Sex hormones affect the structure of the renal tubules and various aspects of renal functions in experimental animals and humans. Most of today's knowledge is based on studies in rodents showing that sex-dependent phenomena in kidneys take place at the level of mRNA and/or protein expression of various transporters in the apical and basolateral membranes of the cells in specific parts of the nephron [31].

The limitation of this study should be considered in the light of the results. The study was cross-sectional, and therefore patients with decreased eGFR were identified retrospectively.

\section{Conclusions}

In conclusion, MAP was associated with decreased eGFR in men, while in women MAP was not associated with decreased eGFR. These findings provide some evidence that a different adaptive response to renal regulation may exist in males and females, and paves the way for further studies aiming to identify the sex hormones involved in this mechanism.

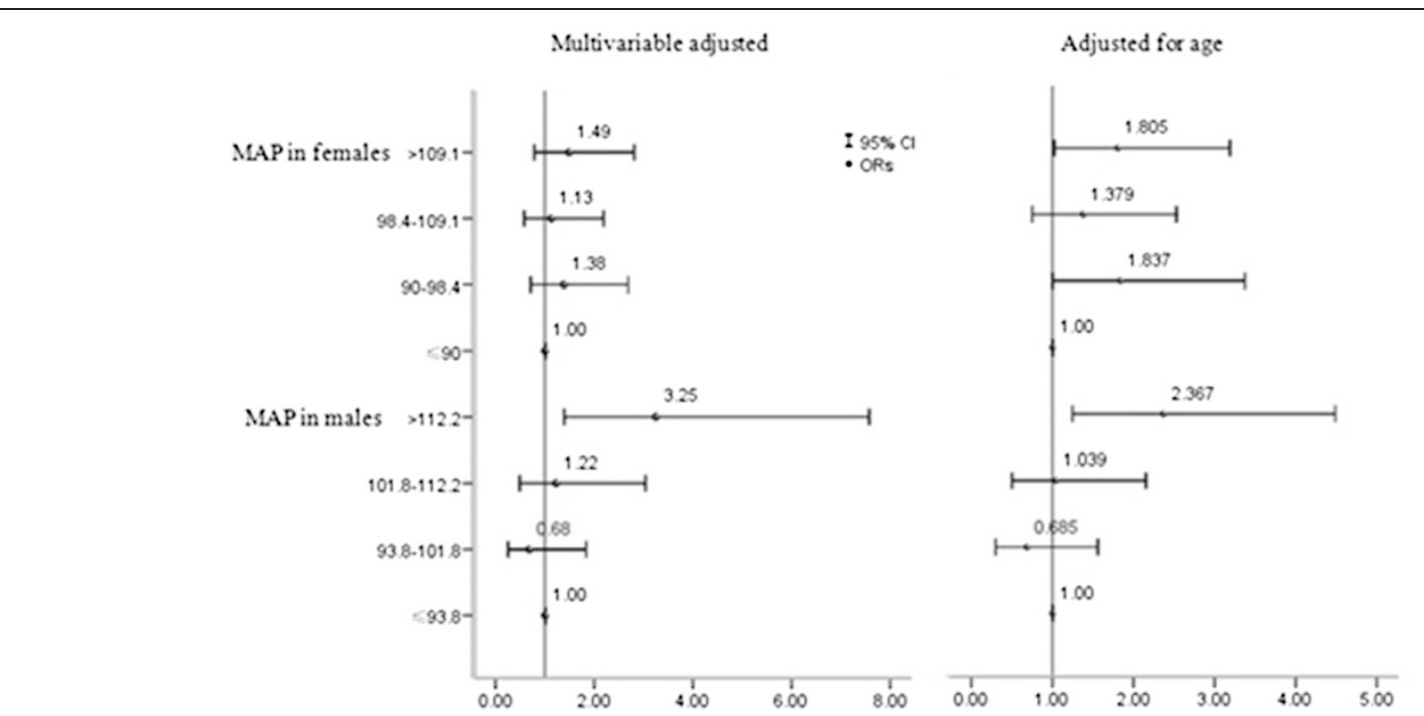

Fig. 2 The OR and $95 \% \mathrm{Cl}$ for the prevalence of decreased eGFR as a function of quartiles of MAP. Multivariable analysis adjusted for age, BMI, WC, mean SBP, mean DBP, mean PP, smoking status, drinking status, ethnic group, education, income, history of stroke, history of heart disease, total cholesterol, triglyceride, LDL-C, HDL-C, fasting glucose, hemoglobin, and uric acid 


\section{Abbreviations}

eGFR: Glomerular filtration rate; CVD: Cardiovascular disease; MAP: Mean arterial pressure; PP: Pluse pressure; BP: Blood pressure; RBF: Low renal blood flow; BMl: Body mass index; WC: Waist circumference; FPG: Fasting plasma glucose; TC: Total cholesterol; LDL-C: Low-density lipoprotein cholesterol; HDL-C: High-density lipoprotein cholesterol; TG: Triglyceride; SBP: Systolic BP; DBP: Diastolic BP; WHO: World Health Organization; OR: Odds ratio; $\mathrm{Cl}$ : Confidence interval.

\section{Competing interests}

The authors declare that they have no competing interests.

\section{Authors' contributions}

HMY carried out study design, performed the epidemiological survey and data input, analysed data. YXS conceived and carried out study design, XFG, $X G Z, Z L, S S Y, W N L$ and YZ performed the epidemiological survey and data input. XFG, LQZ, analysed data. All authors were involved in writing the paper and had final approval of the submitted and published versions.

\section{Acknowledgements}

This study was supported by grants from the "Twelfth Five-Year" project funds (National Science and Technology Support Program of China, Grant No. 2012BAJ18B02).

\section{Author details}

${ }^{1}$ Department of Cardiology, The First Hospital of China Medical University, 155 Nanjing North Street, Heping District, Shenyang 110001, Liaoning, China. ${ }^{2}$ Department of Clinical Epidemiology, Library, Shengjing Hospital of China Medical University, Shenyang, Liaoning, China.

Received: 12 December 2014 Accepted: 20 July 2015 Published online: 13 August 2015

\section{References}

1. Muntner P, He J, Hamm L, Loria C, Whelton PK. Renal insufficiency and subsequent death resulting from cardiovascular disease in the United States. J Am Soc Nephrol. 2002;13(3):745-53.

2. Amann K, Tyralla K. Cardiovascular changes in chronic renal failure: pathogenesis and therapy. Clin Nephrol. 2002;58 Suppl 1:S62-72.

3. Verdecchia P, Schillaci G, Reboldi G, Franklin SS, Porcellati C. Different prognostic impact of 24-hour mean blood pressure and pulse pressure on stroke and coronary artery disease in essential hypertension. Circulation. 2001;103:2579-84

4. Langenberg C, Bellomo R, May C, Wan L, Egi M, Morgera S. Renal blood flow in sepsis. Crit Care. 2005;9:R363-74.

5. LeDoux D, Astiz ME, Carpati CM, Rackow EC. Effects of perfusion pressure on tissue perfusion in septic shock. Crit Care Med. 2000;28:2729-32.

6. Bourgoin A, Leone M, Delmas A, Garnier F, Albanese J, Martin C. Increasing mean arterial pressure in patients with septic shock: effects on oxygen variables and renal function. Crit Care Med. 2005:33:780-6.

7. Deruddre S, Cheisson G, Mazoit JX, Vicaut E, Benhamou D, Duranteau J. Renal arterial resistance in septic shock: effects of increasing mean arterial pressure with norepinephrine on the renal resistive index assessed with Doppler ultrasonography. Intensive Care Med. 2007;33:1557-62.

8. Chue CD, Edwards NC, Davis $\sqcup$, Steeds RP, Townend JN, Ferro CJ. Serum phosphate but not pulse wave velocity predicts decline in renal function in patients with early chronic kidney disease. Nephrol Dial Transplant. 2011;26:2576-82

9. Upadhyay A, Hwang SJ, Mitchell GF, Vasan RS, Vita JA, Stantchev PI, et al. Arterial stiffness in mild-to-moderate CKD. J Am Soc Nephrol. 2009;20:2044-53.

10. Eddington H, Sinha S, Li E, Hegarty J, Ting J, Lane B, et al. Factors associated with vascular stiffness: cross-sectional analysis from the Chronic Renal Insufficiency Standards Implementation Study. Nephron Clin Pract. 2009;112:c190-8.

11. Matsui Y, Ishikawa J, Shibasaki S, Shimada K, Kario K. Association between home arterial stiffness index and target organ damage in hypertension: comparison with pulse wave velocity and augmentation index. Atherosclerosis. 2011;219:637-42.

12. Dunser MW, Ruokonen E, Pettila V, Ulmer H, Torgersen C, Schmittinger CA, et al. Association of arterial blood pressure and vasopressor load with septic shock mortality: a post hoc analysis of a multicenter trial. Crit Care. 2009;13:R181.

13. O'Brien E, Petrie J, Littler W, de Swiet M, Padfield PL, O'Malley K, et al. The British Hypertension Society protocol for the evaluation of automated and semi-automated blood pressure measuring devices with special reference to ambulatory systems. J Hypertens. 1990:8:607-19.

14. James PA, Oparil S, Carter BL, Cushman WC, Dennison-Himmelfarb C, Handler J, et al. 2014 evidence-based guideline for the management of high blood pressure in adults: report from the panel members appointed to the Eighth Joint National Committee (JNC 8). JAMA. 2014;311:507-20.

15. World Health Organization. Hypertension control: report of a WHO Expert Committee. World Health Organ Tech Rep Ser. 1996;862:1-83.

16. WHO/FAO. Expert consultation on diet, nutrition and the prevention of chronic diseases, Report of the Joint WHO/FAO Expert Consultation. Geneva: World Health Organization; 2003.

17. Expert Panel on Detection, Evaluation, and Treatment of High Blood Cholesterol in Adults. Executive summary of the third report of the National Cholesterol Education Program (NCEP) expert panel on detection, evaluation, and treatment of high blood cholesterol in adults (Adult Treatment Panel III). JAMA. 2001;285:2486-97.

18. World Health Organization and International Diabetes Fedaration. Definition and diagnosis of diabetes mellitus and intermediate hyperglycemia: report of a WHO/IDF consultation. Geneva, Switzerland: World Health Organization; 2006.

19. Zhang L, Wang F, Wang L, Wang W, Liu B, Liu J, et al. Prevalence of chronic kidney disease in China: a cross-sectional survey. Lancet. 2012;379:815-22.

20. World Health Organization. Nutritional Anemia: Report of a WHO Scientific Group. Geneva, Switzerland: World Health Organization; 1968.

21. Hu G, Tuomilehto J, Silventoinen $K$, Barengo N, Jousilahti P. Joint effects of physical activity, body mass index, waist circumference and waist-to-hip ratio with the risk of cardiovascular disease among middle-aged Finnish men and women. Eur Heart J. 2004;25:2212-9.

22. Levey AS, Stevens LA, Schmid CH, Zhang YL, Castro 3rd AF, Feldman HI, et al. A new equation to estimate glomerular filtration rate. Ann Intern Med. 2009:150:604-12.

23. Levey AS, Bosch JP, Lewis JB, Greene T, Rogers N, Roth D. A more accurate method to estimate glomerular filtration rate from serum creatinine: a new prediction equation. Modification of Diet in Renal Disease Study Group. Ann Intern Med. 1999;130:461-70.

24. Franklin SS, Gustin WIV, Wong ND, Larson MG, Weber MA, Kannel WB, et al. Hemodynamic patterns of age-related changes in blood pressure: the Framingham Heart Study. Circulation. 1997;96:308-15.

25. Yongbin NI, Wang $\mathrm{H}$, Dayi HU, Zhang W. The relationship between pulse wave velocity and pulse pressure in Chinese patients with essential hypertension. Hypertens Res. 2003;26:871-4.

26. Benetos A, Laurent S, Asmar RG, Lacolley P. Large artery stiffness in hypertension. J Hypertens. 1997;15(suppl):S89-97.

27. Liu YL, Prowle J, Licari E, Uchino S, Bellomo R. Changes in blood pressure before the development of nosocomial acute kidney injury. Nephrol Dial Transplant. 2009;24:504-11.

28. Kummer S, von Gersdorff G, Kemper MJ. The influence of gender and sexual hormones on incidence and outcome of chronic kidney disease. Pediatr Nephrol. 2012;27:1213-9.

29. Musselman TM, Zhang Z, Masilamani SM. Differential regulation of the bumetanide-sensitive cotransporter (NKCC2) by ovarian hormones. Steroids. 2010;75(11):760-5.

30. Amorim MZ, Machado M. Hackney Sex differences in serum ck activity but not in glomerular filtration rate after resistance exercise: is there a sex dependent renal adaptative response? J Physiol Sci. 2014;64:31-6.

31. Sabolić I, Asif AR, Budach WE, Wanke C, Bahn A, Burckhardt G. Gender differences in kidney function. Pflugers Arch. 2007:455:397-429. 